Received: 31.08 .2018

Revised: 25.12 .2018

Accepted: 15.02 .2019

DOI: $10.17804 / 2410-9908.2019 .1 .064-069$

\title{
VARIATIONAL PRINCIPLE FOR THE VELOCITIES OF PARTICLES OF A VISCOPLASTIC STRIP UNDER ROLLING
}

\author{
V. D. Solovei \\ Institute of Engineering Science, Ural Branch of the Russian Academy of Sciences, \\ 34 Komsomolskaya St., 620049, Ekaterinburg, Russian Federation \\ (iD https://orcid.org/0000-0003-4696-8706 \$ SoloveiVD@yandex.ru \\ Corresponding author: E-mail: SoloveiVD@yandex.ru \\ Address for correspondence: ul. Komsomolskaya, 34, 620049, Ekaterinburg, Russian Federation \\ Tel.: +7 (343) 37535 92; fax: +7 (343) 3745330
}

Plane flow of a viscoplastic strip under rolling is considered. Tangential friction stresses at the flow-roll interface and at contact of the flow region with the rigid strip ends are specified approximately by the Prandtl friction law. A variational principle is proved for particle velocities with account of the convection flow.

Keywords: viscoplastic strip rolling, stationary flow of a strip, convective flow, variational principle, local potential.

\section{Acknowledgment}

The work was carried out within the framework of the state assignment, theme No. 03912016-0001.

\section{References}

1. Ilyushin A.A. The Deformation of a Visco-Plastic Solid. Uchenye Zapiski Mosk. Gos. Univ., Ser. 2, 1940, vol. 39, pp. 3-81. (In Russian).

2. Mosolov P.P., Miasnikov V.P. Variational methods in the theory of the fluidity of a viscousplastic medium. Journal of Applied Mathematics and Mechanics, 1965, vol. 29, no. 3, pp. 545-577. DOI: 10.1016/0021-8928(65)90063-8.

3. Freydental A., Geyringer Kh. Matematicheskie teorii neuprugoy sploshnoy sredy [Freudenthal Alfred M., Geiringer Hilda. The Mathematical Theories of the Inelastic Continuum. In: Handbuch der Physik, Bd.VI, Berlin, Göttingen, Heidelberg, Springer-Verlag, 1958, pp. 229-433]. Moscow, Fizmatgiz Publ., 1962, 432 p. (In Russian).

4. Kolmogorov V.L. Mekhanika obrabotki metallov davleniem [Mechanics of metal forming]. Moscow, Metallurgiya Publ., 1986, 688 p. (In Russian).

5. Alekseev A.E. Nonlinear laws of dry friction in contact problems of linear theory of elasticity. Journal of Applied Mechanics and Technical Physics, 2002, vol. 43, iss. 4, pp. 622-629. DOI: 10.1023/A:1016018118184. 
Подана в журнал: 31.08 .2018

УДК 539.3

DOI: $10.17804 / 2410-9908.2019 .1 .064-069$

\title{
ВАРИАЦИОННЫЙ ПРИНЦИП ДЛЯ СКОРОСТЕЙ ЧАСТИЦ ВЯЗКОПЛАСТИЧЕСКОЙ ПОЛОСЫ ПРИ ПРОКАТКЕ
}

\author{
В. Д. Соловей \\ Федеральное государственное бюджетное учреждение науки \\ Институт машиноведения Уральского отделения Российской академии наук, \\ ул. Комсомольская, 34, Екатеринбург, Российская Федераџия \\ (iD https://orcid.org/0000-0003-4696-8706 \SoloveiVD@yandex.ru \\ Ответственный автор. Электронная почта: soloveivd@yandex.ru \\ Адрес для переписки: 620049, Екатеринбург, ул. Комсомольская, 34, Российская Федерация \\ Тел.: +7 (343) 375-35-92; факс: +7 (343) 374-53-30
}

Рассматривается плоское течение вязкопластической полосы при прокатке. Касательные напряжения трения на границах контакта области течения с валком и с жесткими концами полосы задаются приближенно с помощью закона трения Прандтля. Доказан вариационный принцип для скоростей частиц с учетом конвективного течения.

Ключевые слова: прокатка вязкопластической полосы, стационарное течение полосы, конвективное течение, вариационный принцип, локальный потенциал.

\section{1. Введение}

В работе [1] представлен вариационный принцип для скоростей частиц вязкопластического тела, на границе которого задаются скорости частиц. Вариационный принцип для краевой задачи течения вязкопластического тела в случае, когда на границе тела задаются скорости частиц и напряжения, рассмотрен в работе [2]. В перечисленных работах не учитывалось конвективное течение материала.

В настоящей работе доказывается вариационный принцип для скоростей частиц вязкопластической полосы, на границе области, течения которой задаются скорости частиц и напряжения, при прокатке с учетом конвективного течения материала. Соответствующий функционал является локальным потенциалом.

\section{2. Краевая задача течения вязкопластической полосы при прокатке}

Система уравнений для стационарного течения вязкопластического тела имеет вид [3, 4]:

$$
\begin{gathered}
\sigma_{i j, j}=\rho w_{i}, \\
v_{i, i}=0, \\
s_{i j}=2 \mu \varepsilon_{i j}+2 K \varepsilon_{i j} / H,
\end{gathered}
$$




$$
\begin{gathered}
\varepsilon_{i j}=\left(v_{i, j}+v_{j, i}\right) / 2, \\
\sigma_{i j}=s_{i j}+\sigma \delta_{i j} .
\end{gathered}
$$

Здесь $v_{i}$ и $w_{i}$ компоненты векторов скоростей и ускорений частиц; $\varepsilon_{i j}-$ компоненты тензора скорости деформации; $H=\sqrt{2 \varepsilon_{i j} \varepsilon_{i j}}-$ интенсивность скоростей деформации сдвига; $\sigma_{i j}$ и $s_{i j}-$ компоненты тензора напряжений и девиатора напряжений; $\sigma=\sigma_{i i} / 3-$ гидростатическое давление; $\rho$ - плотность тела $(\rho=$ const $) ; \mu$ - коэффициент вязкости; $K$ - предел текучести на сдвиг; $\delta_{i j}$ - символ кронекера; тензорные индексы принимают значения прямоугольных декартовых координат $x, y$; по дважды повторяющимся тензорным индексам $i, j, k, l$ происходит суммирование по всем возможным их значениям; запятая перед индексом обозначает частную производную по соответствующей координате или по времени.

Ускорение

$$
w_{i}=v_{i, j} v_{j}
$$

связано с конвективным движением. функции

Уравнения состояния (3) получены в предположении, что плотность диссипативной

$$
f=s_{i j} \varepsilon_{i j}=\mu H^{2} / 2+K H
$$

является вязкопластическим потенциалом [1, 3]:

$$
s_{i j}=\partial f / \partial \varepsilon_{i j}
$$

Напряжения на границе области деформации полосы определяются формулой

$$
p_{i}=\sigma_{i j} n_{j}
$$

На рисунке введены следующие обозначения: $S$ (область $A B D E F$ ) - область течения полосы; $N$ - нейтральная точка на границе контакта полосы и валка $l_{s 2} ; l_{s 2}^{\prime}$ и $l_{s 2}^{\prime \prime}-$ зоны отставания и опережения на $l_{s 2} ; l_{v 1}$ и $l_{v 2}$ - границы области течения с жесткими концами полосы $S_{1}$ и $S_{2} ; V_{1}$ и $V_{2}$ - скорости перемещений жестких концов полосы; $l_{p}$ - свободная граница; $l_{s 1}$ - ось симметрии процесса прокатки; индексы компонент векторов $t$ и $n$ относятся к локальной системе координат $\vec{t}, \vec{n}$. 


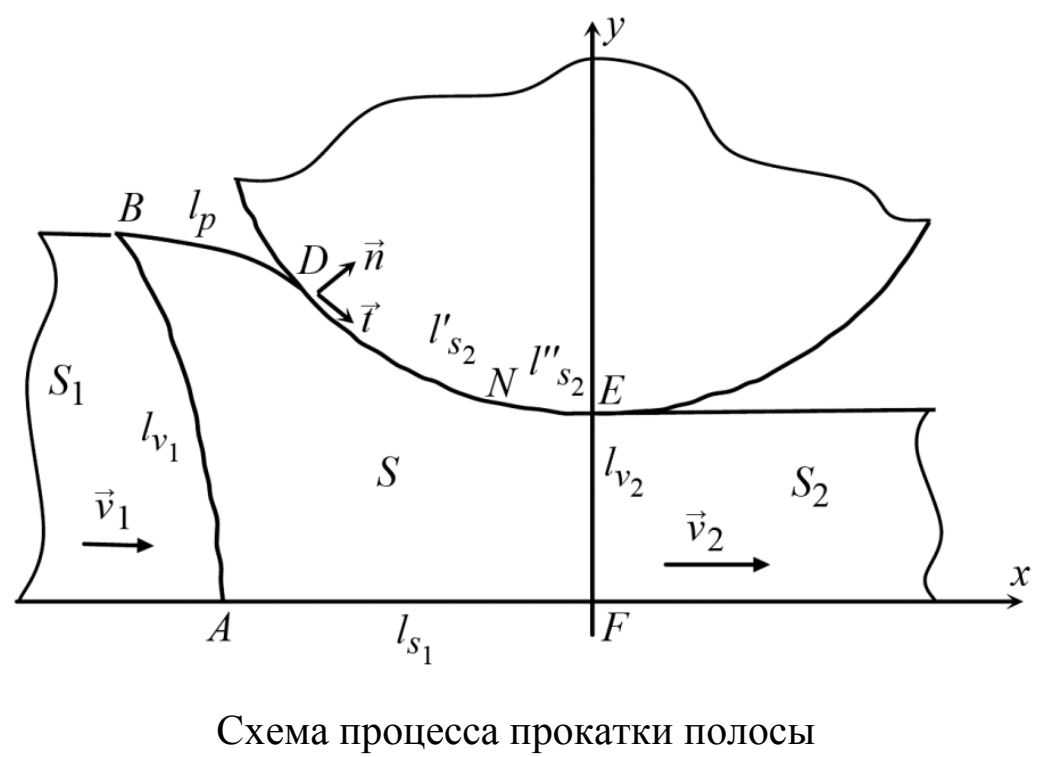

Граничные условия для стационарного течения полосы имеют следующий вид:

$$
\begin{gathered}
p_{t}^{*}=K \text { на } l_{s 2}^{\prime}, p_{t}^{*}=-K \text { на } l_{s 2}^{\prime \prime}, v_{n}^{*}=0 \text { на } l_{s 2} ; \\
\left|p_{t}^{*}\right|=K, v_{n}^{*}=v_{n}^{*}\left(V_{1}\right) \text { на } l_{v 1} ;\left|p_{t}^{*}\right|=K, v_{n}=V_{2} \text { на } l_{v 2} ; \\
p_{t}^{*}=0, \quad p_{n}^{*}=0 \text { на } l_{p} ; p_{t}^{*}=0, \quad v_{n}^{*}=0 \text { на } l_{s 1} .
\end{gathered}
$$

Касательные напряжения трения $p_{t}$ на $l_{s 2}, l_{v 1}$ и $l_{v 2}$ задаются приближенно с помощью закона трения Прандтля [5]. Напряжения трения на $l_{v 1}$ и $l_{v 2}$ являются пассивными. Звездочкой* обозначаются заданные величины.

\section{3. Доказательство вариационного принципа для скоростей частиц вязкопластической полосы при прокатке}

Покажем, что для вязкопластической полосы при прокатке функционал

$$
J=\iint_{S}\left(\mu H^{\prime 2} / 2+K H^{\prime}+\rho w_{i} v_{i}^{\prime}\right) d S-\int_{l_{s 2}} p_{t}^{*} v_{t}^{\prime} d l-\int_{l_{s v 1}} p_{t}^{*} v_{t}^{\prime} d l-\int_{l_{s v 2}} p_{t}^{*} v_{t}^{\prime} d l,
$$

определенный на кинематически возможных скоростях частиц $v_{i}^{\prime}$, удовлетворяющих уравнению (2) и граничным условиям (10)-(12), для действительных скоростей частиц $v_{i}$ принимает минимальное значение. Отметим, что ускорение $w_{i}$ в (13) связано с действительными скоростями частиц $v_{i}$.

Рассмотрим первую вариацию функционала (13), связанную с вариациями скоростей частиц $\delta v_{i}$ относительно действительных скоростей частиц $v_{i}$. 
Учитывая соотношения (8), (5), (2), (4), симметричность тензора напряжений, используя теорему Гаусса-Остроградского, принимая во внимание (9), получим следующее соотношение:

$$
\delta \iint_{S}\left(\mu H^{2} / 2+K H^{\prime}\right) d S=-\iint_{S} \sigma_{i j, j} \delta v_{i} d S+\oint_{l} p_{i} \delta v_{i} d l
$$

Второй интеграл в (14) берется по всей границе области течения $l$.

Первую вариацию функционала (13) с учетом (14), (6), а также граничных условий (10)-(12), преобразуем к следующему виду:

$$
\delta J=\iint_{S}\left(-\sigma_{i j, j}+\rho w_{i}\right) \delta v_{i} d S .
$$

Из (15) и (1) следует равенство

$$
\delta J=0
$$

Покажем, что для второй вариации функционала (13) в действительном состоянии имеет место неравенство

$$
\delta^{2} J>0
$$

Используя (3), можно получить соотношение

$$
\delta^{2}\left(s_{i j} \varepsilon_{i j}\right)=4 K H^{-3}\left(\varepsilon_{i j} \varepsilon_{i j} \delta \varepsilon_{k l} \delta \varepsilon_{k l}-\varepsilon_{i j} \delta \varepsilon_{i j} \varepsilon_{k l} \delta \varepsilon_{k l}\right)+4 \mu \delta \varepsilon_{i j} \delta \varepsilon_{i j} .
$$

Так как выражение в скобках, согласно неравенству Коши-Буняковского, неотрицательно, то для $\delta \varepsilon_{i j} \neq 0$ с учетом (7) и (13) имеет место неравенство (17).

Из (16) и (17) следует минимальность функционала (13) для действительных скоростей перемещения $v_{i}$.

\section{4. Заключение}

Рассматривается плоское течение вязкопластической полосы при прокатке. Касательные напряжения трения на границах контакта области течения с валком и с жесткими концами полосы задаются с помощью закона Прандтля. Вариационный принцип для скоростей частиц доказывается с учетом конвективного движения. Функционал принципа является локальным потенциалом.

\section{Благодарность}

Работа выполнена в рамках государственного задания, тема № 0391-2016-0001.

\section{Список литературы}

1. Ильюшин А. А. Деформация вязко-пластичного тела // ученые записки МГУ. Механика. - 1940. - Вып. 39. - С. 3-81.

2. Mosolov P. P., Miasnikov V. P. Variational methods in the theory of the fluidity of a viscous-plastic medium // Journal of Applied Mathematics and Mechanics. - 1965. - Vol. 29, no. 3. P. 545-577. -DOI: 10.1016/0021-8928(65)90063-8. 
3. Фрейденталь А., Гейрингер Х. Математические теории неупругой сплошной среды. М. : Физматгиз, 1962.

4. Колмогоров В. Л. Механика обработки металлов давлением. - М. : Металлургия, 1986. 5. Alekseev A. E. Nonlinear laws of dry friction in contact problems of linear theory of elasticity // Journal of Applied Mechanics and Technical Physics. - 2002. - Vol. 43, iss. 4. - P. 622-629. DOI: $10.1023 / \mathrm{A}: 1016018118184$. 